\title{
Prevalence and correlates of adverse childhood experiences among adolescents living with HIV-AIDS in Lagos, Nigeria
}

\author{
Suraju Adeyemo', Increase Adeosun², Oluyemi Ogun', Abosede Adegbohun' \\ 'Federal Neuro-Psychiatric Hospital Yaba, Lagos, Nigeria \\ ${ }^{2}$ Mental Health Unit, Department of Internal Medicine, Babcock University/Babcock University Teaching Hospital, llishan-Remo, Nigeria
}

\begin{abstract}
Objective: Adverse childhood experience is a known risk factor for risky behavior and negative mental health outcomes. There is dearth of information on adverse childhood experiences among adolescents living with HIV-AIDS in Nigeria. This study assessed the prevalence, pattern and correlates of adverse childhood experience among adolescents with HIV/AIDS attending two tertiary health facilities in Lagos, Nigeria.
\end{abstract}

Method: Using a cross-sectional study design, 201 adolescents with HIV-AIDS attending out-patient clinics at the Nigerian Institute of Medical Research, Yaba, Lagos and the Lagos state University Teaching Hospital completed the adverse childhood experience questionnaire (ACE) and a socio-demographic questionnaire. The data was analysed with SPSS version 20.
Result: The mean age of the participants was $13.88( \pm 2.53)$ years, and $61.7 \%$ were males. High levels of adverse childhood experiences (ACE $>4$ ) were reported by $28.4 \%$ of the participants. Nearly three quarters $(73.6 \%)$ were victims of physical abuse, while $48.7 \%$ and $11.8 \%$ had been emotionally and sexually abused. There was statistically significant association between high levels of adverse experiences in childhood and occupational class of the father $(p=0.022)$, previous history of hospitalisation $(\mathrm{p}=0.027)$ and having HIV positive siblings $(\mathrm{p}=0.009)$.

Conclusion: There is a crucial need for interventions targeted towards prevention of physical, emotional and sexual child abuse, and other forms of adverse childhood experiences.

Keywords: HIV-AIDS, adverse childhood experiences, child abuse, adolescents

Corresponding author: Dr Suraju Adeyemo, Federal Neuro-Psychiatric Hospital Yaba, Lagos, Nigeria. Email: surajuadeyemo@gmail.com,

\section{INTRODUCTION}

The Joint United Nations Program on HIV/AIDS (UNAIDS) documented in 2005 that $0.6 \%$ of the world population was infected with HIV and declared it as the worst pandemic in human history1. About 78 million people have been infected with HIV and about 35 million lives lost to AIDS-related illnesses since its discovery over 35 years ago2,3. The global summary of the HIV/AIDS epidemic by UNAIDS in 2015 reported that an average of 36.7 million people were living with HIV/AIDS, out of which about 33.4 million were adult and 3.3 million were children or adolescents. According to UNAIDS, the current prevalence of HIV among adults in Nigeria was estimated to be about $3.6 \%{ }^{4,5}$ and $5.2 \%$ among adolescents ${ }^{5}$.

The World Health Organization classified adolescence as the period between the ages of 10 and 19 years. Adolescence is a period associated with many changes which range from biological changes such as puberty, social and psychological changes associated with awareness of sexuality ${ }^{6-8 .}$ The HIV/AIDS epidemic in adolescents is peculiar because they are faced with diverse problems ranging from adverse childhood experience to difficulties accepting their HIV status and the need for lifelong treatment 8. During adolescence, individuals with HIV/AIDS are more likely to witness the death of their parent and more prone to physical, emotional and sexual abuse. 9

Adverse childhood experiences are one of the common important modifiable psychosocial causes of co-morbidity in adolescents with HIV/AIDS10,11. Physical, emotional, family dysfunction and sexual abuse have been reported to be more prevalent among people living with HIV/AIDS compared to the general population $10,12,13$. Studies have shown that adverse childhood experiences contribute to poor adherence to treatment, repeated emergency visits and hospitalisation, worsen the progression of HIV infection, and increases risks of morbidity and mortality10,12,14 ${ }^{15}$. Adverse childhood experience has also been associated with risky sexual behavior, substance abuse, poor coping styles, engagement in health care system, and poor adherence to treatment $10^{, 13,14}$.

Adverse childhood experiences tend to occur in clusters. Consequently, the presence of an adverse childhood experience may be an indication that the individual could be experiencing other forms of child abuse $16^{-19}$. It has also been shown that 
different forms of adversities experienced in childhood have cumulative effect in adulthood, which could be proportional to the intensity of the adversities the individual has been exposed to before the age of $189^{18-20}$.

Available data on adverse childhood experiences in HIV/AIDS were obtained from studies conducted in other parts of the world. There is dearth of information on this subject in Nigeria and the West African region. The current study therefore aimed to assess the prevalence and pattern of adverse childhood experience among adolescents living with HIV/AIDS. Secondarily, the association between adverse childhood experiences, and socio-demographic and HIVrelated characteristics of the participants were determined.

\section{METHODS}

Study design and setting: The study was a descriptive cross-sectional study carried out among 201 adolescents with HIV aged 10-19 years attending the outpatient clinic of the Pediatric/Adult Hematology unit of Lagos state University Teaching Hospital and the Nigerian Institute of Medical Research, Yaba, Lagos, Nigeria. Both study locations were urban settings within the Lagos metropolis.

Ethical considerations: Ethical approval was obtained from the research ethics committees of the Lagos State University Teaching Hospital and the Nigerian Institute of Medical Research. A signed consent form was taken from the parent/caregiver and a verbal assent from the adolescents aged less than 18 years while an informed consent was taken from adolescents aged 18-19 years after they had been adequately informed about the nature, extent, and purpose of the study. All the participants had knowledge of their HIV status prior to their participation in the study.

\section{Sample size determination:}

This was computed using the Fisher's formula $\mathrm{n}=$ $\mathrm{z}^{2} \mathrm{pq} / \mathrm{d}^{2}$

Where $\mathrm{n}=$ the desired sample size, $\mathrm{z}=$ the standard normal deviation set at 1.96 which corresponds to the $95 \%$ confidence level; $p=$ the proportion in the target population estimated to have a particular characteristic, taken to be $40.8 \%$ (i.e. 0.41 ); $\mathrm{q}=1.0$ $\mathrm{p}$ (1-0.41); $\mathrm{d}=$ degree of accuracy desired, set at

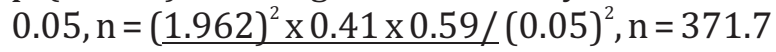

Because of the small population size $(\mathrm{N}=360)$, a sample size correction was done using Finite Population Correction for Proportions;

$$
\mathrm{n}=\frac{\text { no }}{1+(\text { no }-1)}
$$

no $=$ sample size $=372 ; \mathrm{N}=$ population size $=$ adolescents attending HIV outpatient clinic in LASUTH and NIMR $=360$

$\mathrm{n}=183$. Additional $10 \%$ was included to cater for attrition rate $(183+18.3=201.3)$

\section{Sampling Technique:}

Adolescents who met the inclusion criteria for the study were consecutively recruited into the study until the required sample size was obtained.

Inclusion and Exclusion Criteria:

The inclusion criteria consisted of adolescents with laboratory confirmed diagnosis of HIV, with consent to participate in the study signed by the caregiver, and assent given by the adolescent while those with other comorbid chronic medical conditions and those who were too acutely ill to be interviewed were excluded from the study.

\section{Study Instruments:}

The adverse childhood experience (ACE) questionnaire: This is a self report questionnaire developed in San Diego, California in 1995 by Felitti et al to assess four major categories of adverse childhood experience namely physical abuse, emotional/psychological abuse, sexual abuse and family dysfunction21. The ACE uses a simple scoring method to determine the extent of exposure to childhood trauma. The responses are in "Yes" or "No" format, a "yes" response is given a score of one while a "NO" is given zero21. The value derived after adding up all the scores on each response is considered as the ACE score of the individual. For the purpose of this study, a score below four was considered as low while scores of four and above was taken as high

Socio-demographic questionnaire: This was designed to obtain the socio-demographic characteristics of the participants. The information obtained included age, gender, educational level, ethnicity, parent marital status, parental employment status, health related variable like;sources of infection, past history of hospital admissions,HIV status in parent and sibblings.

\section{Procedure}

On each clinic day, adolescents at the HIV clinic who met the inclusion criteria were identified. The study was explained to them and their caregiver(s). The adolescents were further informed that their caregivers may be interviewed to clarify information when necessary.

The number of selected adolescents scheduled to be seen on each clinic day were then given the socio-demographic questionnaire to complete at the waiting room of the clinic, at each study center. Thereafter, each participant was called into the consulting room where the adverse childhood event questionnaire was administered 
by self-report and additional information about HIV-related variables was obtained from their hospital case notes.

\section{Statistical Analysis:}

The Statistical Package for Social Sciences (IBMSPSS) version 20 was used to analyze all collated data. The primary outcome of interest (dependent variable) was dichotomised into high or low adverse childhood experiences based on a cut-off score of 4 on the adverse childhood experiences questionnaire. Descriptive statistics including frequencies, percentages, mean and standard deviation were calculated for relevant sociodemographic and HIV-related characteristics. Bivariate analysis using chi-square determined the association between the outcome variable and selected independent variables.

\section{RESULT}

The ages of the participants ranged from 10-19 years, with a mean age of $13.88( \pm 2.53)$ years (Table1). Early and mid adolescents constituted $47.3 \%$ and $40.3 \%$ respectively of the study participants respectively, while $12.4 \%$ were late adolescents. About six out of every ten (61.7\%) participants were males. Half (50.7\%) of the participant were from the Yoruba ethnic group compared to $25.9 \%$ from Igbo, $7 \%$ from Hausa and $16.4 \%$ from other ethnic group. All the participants were single and students.

The majority (87.1\%) of the adolescents acquired the HIV infection via vertical transmission (at birth). Others contacted the infection through sexual intercourse, blood transfusion and sharing of sharp objects (Table 2).

Table 1: Socio-demographic characteristics of adolescents with HIV-AIDS ( $\mathbf{N}=\mathbf{2 0 1})$

\begin{tabular}{|c|c|c|}
\hline Variables & $\mathbf{n}$ & $\%$ \\
\hline \multicolumn{3}{|l|}{ Age Range (in years) } \\
\hline Early Adolescent (10-13) & 95 & 47.3 \\
\hline Middle Adolescent (14-16) & 81 & 40.3 \\
\hline Late Adolescent (17-19) & 25 & 12.4 \\
\hline \multicolumn{3}{|l|}{ Gender } \\
\hline Male & 124 & 61.7 \\
\hline Female & 77 & 38.3 \\
\hline \multicolumn{3}{|l|}{ Ethnic group } \\
\hline Yoruba & 102 & 50.7 \\
\hline Igbo & 52 & 25.9 \\
\hline Hausa & 14 & 7.0 \\
\hline Other & 33 & 16.4 \\
\hline \multicolumn{3}{|l|}{ Religion } \\
\hline Christianity & 158 & 78.6 \\
\hline Islam & 43 & 21.4 \\
\hline \multicolumn{3}{|l|}{ Level of education } \\
\hline Primary & 38 & 18.9 \\
\hline Junior secondary school & 76 & 37.8 \\
\hline Senior secondary school & 76 & 37.8 \\
\hline Post-secondary school & 11 & 5.5 \\
\hline \multicolumn{3}{|c|}{ Present living circumstances } \\
\hline With both parent & 97 & 48.3 \\
\hline With single parent & 62 & 30.8 \\
\hline With others & 42 & 20.9 \\
\hline \multicolumn{3}{|l|}{ Family type } \\
\hline Monogamous & 137 & 68.2 \\
\hline Polygamous & 64 & 31.8 \\
\hline \multicolumn{3}{|l|}{ Occupational class (Father) } \\
\hline ISCO $1-3$ & 115 & 57.2 \\
\hline ISCO 4-6 & 53 & 26.4 \\
\hline ISCO7-10 & 33 & 16.4 \\
\hline \multicolumn{3}{|l|}{ Occupational class (Mother) } \\
\hline ISCO 1-3 & 55 & 27.4 \\
\hline ISCO 4-6 & 78 & 38.8 \\
\hline ISCO7-10 & 68 & 33.8 \\
\hline
\end{tabular}


Table2: HIV-related characteristics of the participants $(N=201)$

Variables

n

175

26

46

155

103

98

151

50

Negative

HIV status in siblings

Positive

Negative

43

158

More than a quarter $(28.4 \%)$ of the participants had high (>4) score in the adverse childhood experience (ACE) scale (Table 3). Nearly three quarters $(\mathbf{7 3 . 6 \%})$ of the participants had a history
$\%$

87.1

12.9

22.9

77.1

51.2

48.8

75.1

24.9

21.4

78.6

of physical abuse, more than half $(57.2 \%)$ were emotionally abused while $11.4 \%$ had history of sexual abuse. There was history of family dysfunction among $45.3 \%$ of the participants.

Table 3: Pattern of Adverse Childhood Experiences among Adolescents with HIVAIDS

\section{Variables}

Adverse childhood event score

High adverse event score

Low adverse event score

Physical abuse

Yes

No

Emotional abuse

Yes

No

Sexual abuse

Yes

No

Family dysfunction

Yes 
On bivariate analysis, history of hospital admission, HIV status in siblings and occupational class of participants' father were the only factors statistically significantly related to adverse childhood experience in adolescents with HIV (Tables 4 and 5).

Vedrg"6く"Association between socio-demographic characteristics and adverse childhood experiences (ACE)

$\mathbf{N}=\mathbf{2 0 1}$

\section{ACE SCORE}

\begin{tabular}{lrrrrr} 
Variables & $\begin{array}{c}\text { High } \\
\mathbf{n}(\%)\end{array}$ & $\begin{array}{l}\text { Low } \\
\mathbf{n}(\%)\end{array}$ & Total & $\mathbf{X}^{\mathbf{2}}$ & $\mathbf{P}$ \\
\hline Age Range (in years) & & & & & \\
Early Adolescent (10-13) & $27(28.4)$ & $68(71.6)$ & 95 & 0.298 & 0.861 \\
Middle Adolescent (14-16) & $24(29.6)$ & $57(70.4)$ & 81 & & \\
Late Adolescent (17-19) & $6(24.0)$ & $19(76.0)$ & 25 & & \\
Gender & & & & & \\
Male & $38(30.6)$ & $86(69.4)$ & 124 & 0.833 & 0.361 \\
Female & $19(24.7)$ & $58(75.3)$ & 77 & & \\
Ethnic group & & & & & \\
Yoruba & $31(30.4)$ & $71(69.6)$ & 102 & 0.820 & 0.845 \\
Igbo & $15(28.2)$ & $37(71.2)$ & 52 & & \\
Hausa & $3(21.4)$ & $11(78.6)$ & 14 & & \\
Other & $8(24.2)$ & $25(75.8)$ & 33 & & \\
Present living circumstances & & & & & \\
With both parent & $31(32.0)$ & $66(68.0)$ & 97 & 3.604 & 0.165 \\
With single parent & $19(30.6)$ & $43(69.4)$ & 62 & & \\
With others & $7(16.7)$ & $35(83.3)$ & 42 & & \\
Family type & & & & & \\
Monogamous & $38(27.7)$ & $99(72.3)$ & 137 & 0.082 & 0.775 \\
Polygamous & $19(29.7)$ & $45(70.3)$ & 64 & & \\
Occupational class (father) & & & & & \\
ISCO 1-3 & $30(26.1)$ & $85(73.9)$ & 115 & 7.637 & $\mathbf{0 . 0 2 2}$ \\
ISCO 4-6 & $22(41.5)$ & $31(58.5)$ & 53 & & \\
ISCO 7-10 & $5(15.2)$ & $28(84.8)$ & 33 & & \\
Occupational class (mother) & & & & & \\
ISCO 1-3 & $13(23.6)$ & $42(76.4)$ & 55 & 0.864 & 0.649 \\
ISCO 4-6 & $24(30.8)$ & $54(69.2)$ & 78 & & \\
ISCO 7-10 & $20(28.4)$ & $144(71.6)$ & 68 & & \\
\hline & & & & \\
\hline
\end{tabular}


Table 5: Association between HIV-related characteristics and adverse childhood

experiences (ACE)

$\mathbf{N}=\mathbf{2 0 1}$

ACE SCORE

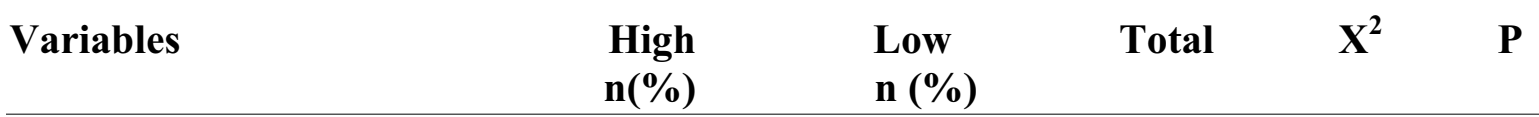

\section{Route of infection}

At birth

Other

Hospital admission due to HIV
Yes

HIV status in father

Positive

Negative

HIV status in mother

Positive

Negative

HIV status in siblings

Positive

Negative
$52(29.7)$

$5(19.2)$

19(41.3)

$38(24.5)$

28(27.2)

29(29.6)

47(31.1)

10(20.0)

19(44.2)

$38(24.1)$

$$
123(70.3)
$$

21(80.8)

27(58.7)

117(75.5)

$75(72.8)$

69(70.4)

104(68.9)

40(80.0)

24(55.8)

$120(75.9)$
175

1.225

0.268

26

46

4.921

$\mathbf{0 . 0 2 7}$

155

103

0.143

0.705

98

151

2.289

0.130

50

43

6.745

0.009

\section{DISCUSSION}

The current study investigated the prevalence, pattern and correlates of adverse childhood experiences among adolescents with HIV in Nigeria. To the best of our knowledge, this subject has not been previously reported in the studied setting. The socio-demographic profile of the participants in our study is consistent with those previously reported by researchers on this subject $22^{-26}$.

The proportion of participants born to polygamous family setting (31.8\%) is remarkably higher than the national average of $4.5 \%$ reported by the Nigerian population commission 27. It is not surprising that less than half $(48.3 \%)$ of the participants live with both parents considering the social disruptions associated with HIV morbidity and mortality.

In the current study, about three quarters of the mothers and half of the fathers of the participants were retro-viral positive. Consequently, the majority (87.1\%) of the participants contacted HIV infection via vertical transmission. Extant evidence has also shown that vertical transmission is the commonest route of transmission of HIV to adolescents4,28. However a study conducted in India found sexual route as the commonest route of HIV transmission in a sample of adolescents $8^{23}$. The preponderance of HIV transmission through sexual intercourse in the Indian study8 could be attributed to the overrepresentation of adolescent sex workers and victims of child trafficking in their sample. The adolescents in the current study were predominantly (96.5\%) compliant with antiretroviral medication, and only a fifth of them have been hospitalised on account of HIV-related morbidity. Adherence with antiretroviral medications has led to a decline in rates of mortality and hospital admission among children and adolescents with HIV infection29.

About 3 out of 10 participants reported a high level of adverse childhood experiences, in the current study. This is in keeping with findings of a previous research on this subject, though majority of previous studies have reported higher rates of adverse childhood experiences ${ }^{24-26,30-33}$. Studies have shown that adolescents with HIV-AIDS are at increased risk of being victims of violence, compared to the general population ${ }^{24-26,32}$.

The risk for child violence victimisation in families affected by HIV-AIDS has been attributed to a number of factors including intimate partner violence, substance abuse, HIV-related stigma and social alienation ${ }^{25}$. Exposure to violence in childhood, in turn, predisposes to use of psychoactive substances, risky sexual behavior and 
increased risk of becoming HIV infected ${ }^{26,32}$.

About three quarters (73.6\%) of the adolescents with HIV-AIDS in the current study have experienced physical abuse, more than half $(57.2 \%)$ had been victims of emotional abuse, and $11.4 \%$ had been sexual abused. Evidences from several studies including meta-analysis and systematic reviews have demonstrated that victims of physical, sexual or emotional abuse have higher risks of engaging in risky sexual behavior, contacting sexually transmitted infections and HIVAIDS $^{32,33}$. Physical and emotional abuse have been linked to poor impulse control, low self esteem and anti-social traits ${ }^{34-36}$. Sexual abuse has also been associated with risky sexual behavior in adolescence including earlier onset of sexual intercourse, unprotected sex, multiple sexual partners, transactional sex and use of alcohol and other psychoactive substances during sex ${ }^{34-36}$.

The pattern of abuse experienced by adolescents with HIV in the current sample differed from that observed among a normal population of adolescents in a national survey conducted by UNICEF in Nigeria ${ }^{37}$. In the UNICEF study, lower prevalence of emotional abuse was reported, and higher rates of sexual abuse ${ }^{37}$.

Children and adolescents with HIV-AIDS may be more vulnerable to emotional abuse than normal population of adolescents due to the stigma associated with the disease. Studies have shown all forms of child abuse have far-reaching consequences on behavioural patterns and emotional well-being later in life, though the mechanism has not been clearly elucidated. Adverse childhood experiences interfere with the development of normal neuro-physiological processes related to stress response, affect regulation and emotional development, with consequent cognitive and psycho-social impairments ${ }^{38}$.

Participants with previous history of hospitalisation and those with HIV positive siblings had significantly higher adverse childhood experience scores. The occupational class of the father was also associated with adverse experiences in childhood.

These findings converge with previous research evidence $20^{39,40}$. Families who have more than one child living with HIV-AIDS are likely to encounter a greater psycho-social burden associated with the disease, which could exacerbate existing risk for adverse childhood experiences ${ }^{38}$. Similarly, poverty or socio-economic disadvantage has been shown to increase the risk of child abuse ${ }^{30,}$ ${ }^{33}$. Other socio-demographic variables investigated were not significantly associated with adverse childhood experiences.
This indicates that experience of childhood adversity among adolescents with HIV was not determined by their gender, age, ethnicity or family type. Consequently, adolescents with HIV-AIDS could benefit from interventions targeted at reducing adverse childhood experiences irrespective of these socio-demographic characteristics ${ }^{41}$. Educating parents and the general populace on the negative implications of child abuse is also recommended ${ }^{41}$.

The current study is limited by its cross-sectional design. Therefore inferences regarding causal relationships between adverse childhood experiences and HIV-AIDS related characteristics cannot be asserted. Furthermore the study was conducted in a sample of adolescents receiving antiretroviral medications in a well-resourced urban setting. This limits extrapolation of the findings from this highly selected sample to other populations of adolescents infected with HIV-AIDS.

\section{Conclusion}

In conclusion, about 3 out of 10 adolescents with HIV-AIDS reported high adverse childhood experiences. Nearly three quarters were victims of physical abuse, while $48.7 \%$ and $11.8 \%$ had been emotionally and sexually abused. There was a significant association between occupational class of the father, previous history of hospitalization, having HIV positive siblings and high adverse experiences in childhood. Our findings highlight the need for interventions directed towards amelioration of adverse childhood experiences. 